\title{
Functional characterization of multiple DICER1 mutations in an adolescent
}

\section{Dear Editor,}

We report the case of an adolescent Caucasian female diagnosed with both multinodular goiter (MNG) and a Sertoli-Leydig cell tumor (SLCT) of the ovary (Fig. 1A). She had, at the age of 11.5 years, lower abdominal pain, but tests were not ordered at this point. At 12 years of age bilateral thyroid nodules were detected and 1 year later a thyroidectomy was performed due to pain and progressive enlargement of nodules. Histopathology showed MNG (i.e., nodular hyperplasia) (Supplementary Figure 1, see section on supplementary data given at the end of this article). Pelvic ultrasound and abdominal computed tomography, undertaken at age 12.5 years because of increasing abdominal discomfort, revealed an ovarian tumor measuring $44 \times 39 \times 56 \mathrm{~mm}$ with solid and cystic components. Alpha-fetoprotein (AFP) was elevated at $43 \mu \mathrm{g} / \mathrm{l}$ (normal values: $<12 \mu \mathrm{g} / \mathrm{l}$ ). Right salpingo-oophorectomy was performed. At this time, the histopathological diagnosis was an adult granulosa cell tumor (GCT) of the ovary (like SLCT, a subtype of sex cord-stromal tumor) with no capsular involvement (Supplementary Figure 2). There was no evidence of lymphadenopathy and no lymph node or omental sampling was performed. Currently, the patient is well, is euthyroid on thyroxine supplementation, and menses are regular.

The patient has a complex past medical history consisting of congenital hyperinsulinism which began shortly after birth and required medical treatment with diaxozide until 24 months of age. She had failure to thrive with recurrent diarrhea. At 25 weeks of age, a jejunostomy Roux-en-Y with button was performed for enteral nutrition. Histology of the small intestinal biopsy demonstrated partial villous atrophy to subtotal villous atrophy (not shown) and she was diagnosed with multiple protein allergies. A low allergen diet was instituted under the supervision of a pediatric immunologist and dietician resulting in improved growth. By 22 months of age, gastrointestinal symptoms had resolved and duodenal and jejunal biopsies were normal with no villous atrophy or abnormal inflammatory cell component. A skin rash distributed over the lower abdomen and legs was noted at 10 months and persisted through childhood. Skin biopsy was consistent with atopic dermatitis.

The reported maternal family history included papillary thyroid cancer $(n=1)$, breast cancer $(n=1)$, and ovarian cancer $(n=2)$ affecting second degree relatives, while a paternal second degree relative died at the age of 51 due to cervical cancer. These diagnoses remain unconfirmed by pathology reports. Autism spectrum disorder has been diagnosed in the patient's brother and the mother and father are well and asymptomatic (Fig. 1A).

Constitutional genetic analyses performed included a comparative genomic hybridization array, which revealed a novel paternally inherited small $(\sim 1.0 \mathrm{Mb})$ terminal deletion of $7 \mathrm{p} 22.3$ of unknown significance. In view of the congenital hyperinsulinism, testing of $A B C C 8$ was undertaken. This identified a paternally inherited novel mutation that mutates a highly conserved residue: c.946G > A; COSM687051; p.G316R, which likely explains this aspect of her medical history (Nessa et al. 2015). Germline genetic testing for PTEN mutations (MPLA and both gDNA and cDNA sequencing of entire coding region, including five common mutations located in the PTEN promoter, PathWest Diagnostic Genomics Laboratory, Nedlands, Western Australia) did not identify any gene variants of clinical significance. The patient was referred for DICER1 genetic analysis. Sanger sequencing of the proband's blood lymphocyte gDNA revealed a novel paternally inherited (Fig. 1 and Supplementary Figure 3) germ-line DICER1 mutation, c.5441C $>\mathrm{T}$. The mutation c. $5441 \mathrm{C}>\mathrm{T}$ is predicted to encode p.S1814L (Fig. 1B and C). The best described function of DICER1 protein is to remove the terminal loop from pre-miRNA to produce mature microRNAs (miRNAs). Mature miRNAs are called

Published by Bioscientifica Ltd. 


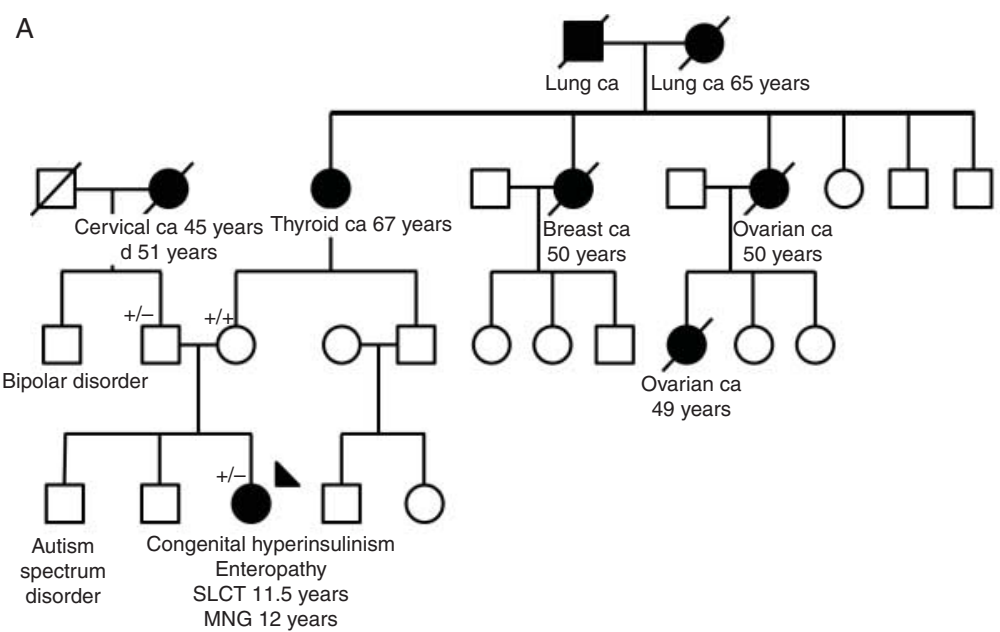

B

C
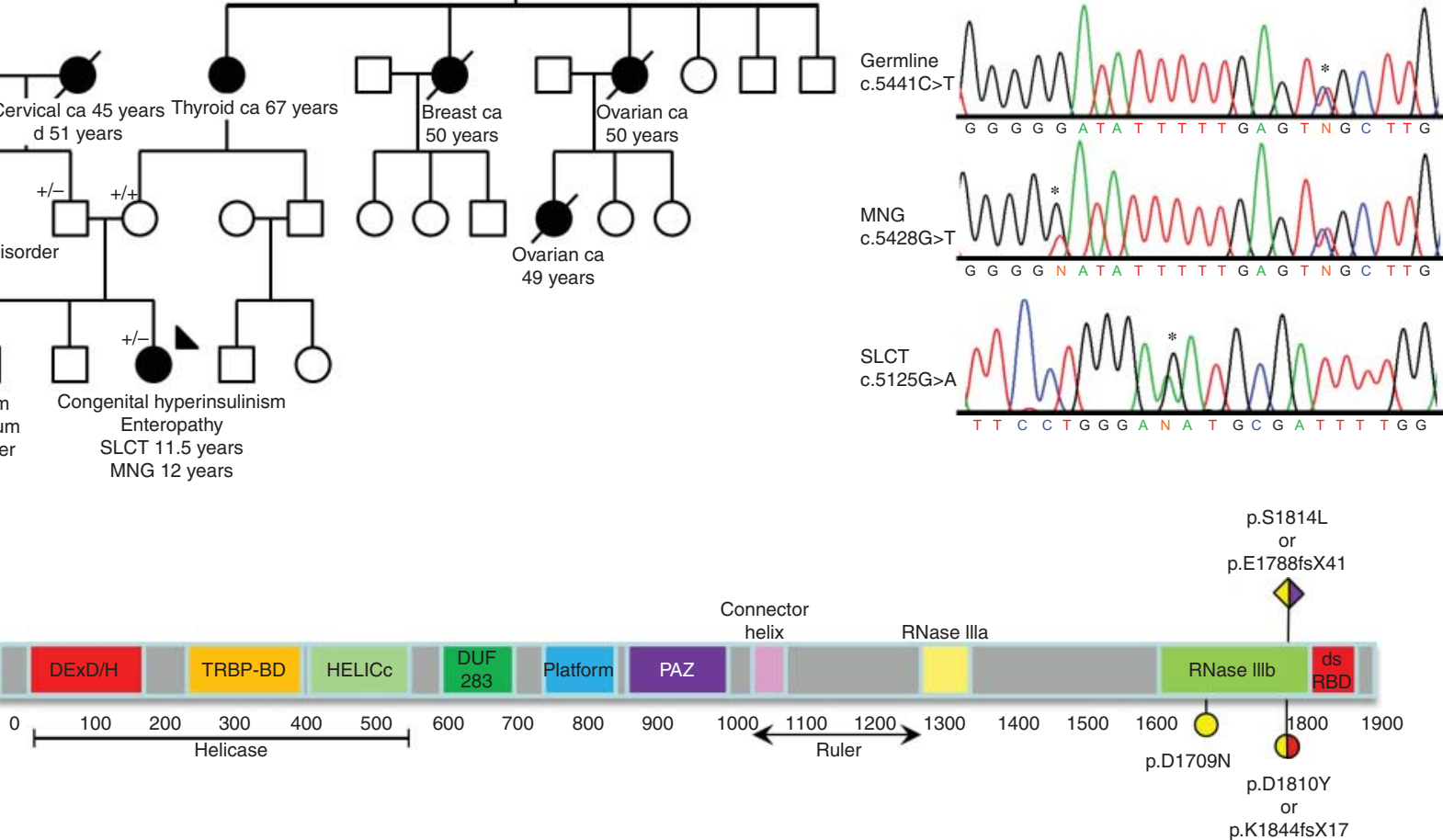

\section{Figure 1}

The proband has germ-line DICER1 mutation c.5441C $>$ T, somatic mutation c.5428G > T in her MNG, and somatic mutation c.5125G > A in her SLCT. (A) Pedigree showing the germ-line mutation was paternally inherited (see Supplementary Figure 3); + / - indicates heterozygosity for the c.5441C $>$ T DICER1 mutation; although the DICER1 mutation was inherited paternally, we note the possibility of a BRCA1 or BRCA2 mutation in the maternal relatives. (B) Sanger sequencing traces showing mutations as marked with *: germ-line mutation c.5441C $>$ T, SLCT somatic mutation c.5125G $>$ A, and MNG somatic mutation c.5428G $>$ T. (C) predicted position of the germ-line

$5 p$ miRNAs if they originate from the 5 prime end or $3 p$ miRNAs if they originate from the 3 prime end of the premiRNA. Typically, germ-line mutations identified in DICER1 are truncating mutations occurring outside of the RNase III domains and are predicted to completely inactivate one copy of the gene (Foulkes et al. 2014). The c.5441C $>$ T missense mutation is doubly unusual in that it occurs within the RNase IIIb domain (Fig. 1C and Supplementary Figure 4) and is not predicted to be truncating. Moreover, S1814 is not one of the critical catalytic (1702 or 1729 (Heravi-Moussavi et al. 2012)) or metal ion binding residues within this domain (these are located at positions 1705, 1709, 1713, 1810, and 1813 (Takeshita et al. 2007)) known to affect processing of $5 \mathrm{p}$ microRNAs (miRNAs). We, therefore, set out to establish the effect of the c.5441C $>\mathrm{T}$ mutation on miRNA processing. and somatic mutations on a linear representation of DICER 1 protein; circles represent somatic mutations, the diamond represents a germline mutation, yellow color (left half of divided shapes and undivided circle) represents missense mutation, purple color (right half of divided diamond) represents deletion created by exon 25 skip, and red color (right half of divided circle) represents the deletion created by the cryptic splice site in exon 25 . SLCT, Sertoli-Leydig cell tumor; MNG, multinodular goiter. A full colour version of this figure is available at http://dx.doi.org/10.1530/ERC-15-0460.

In silico analysis of c. $5441 \mathrm{C}>\mathrm{T}$ predicted that this single base substitution has two likely protein products. One is the aforementioned missense mutation p.S1814L and the other is p.E1788fsX41(abbreviated as p.AEX25), resulting from exon 25 skipping in the transcript (EX-SKIP (Raponi et al. 2011) see ** in Fig. 2A, left panel). Treatment of lymphoblastoid cells from the father with cycloheximide (to inhibit nonsense mediated decay) did not reveal DICER1 transcript lacking exon 25 (Supplementary Figure 5). We employed an in vitro cleavage assay (Supplementary Materials and methods) using radioactively labeled precursor microRNA-122 (pre-miR-122) and FLAG immunoprecipitated p.S1814L, p. $\Delta \mathrm{EX} 25, \mathrm{WT}$, and known $3 p$ and $5 p$ controls to evaluate the functional significance of these two alternate protein products with respect to miRNA biogenesis (see Supplementary Figure 6). Additional variants within the RNAseIIIa/b domains and

Published by Bioscientifica Ltd. 
A

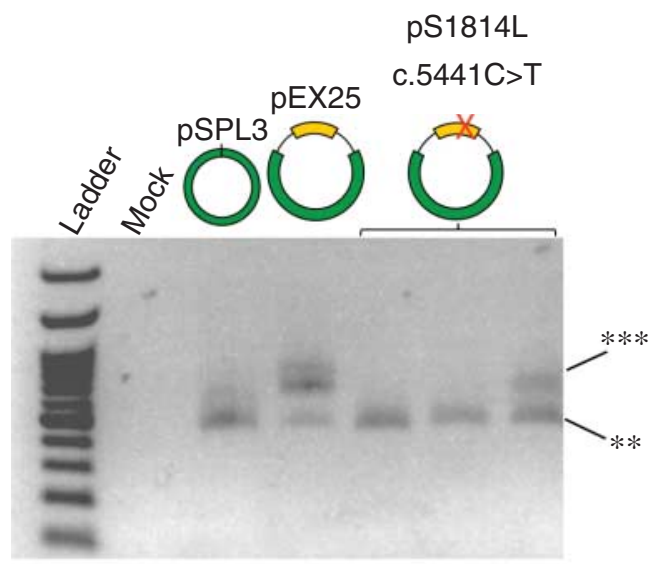

pD1810Y

c. $5428 \mathrm{G}>\mathrm{T}$

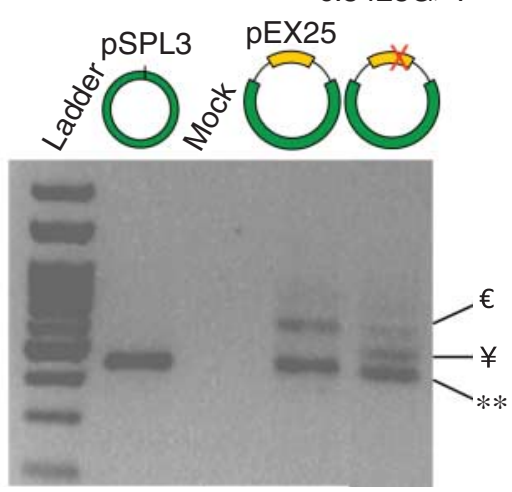

B
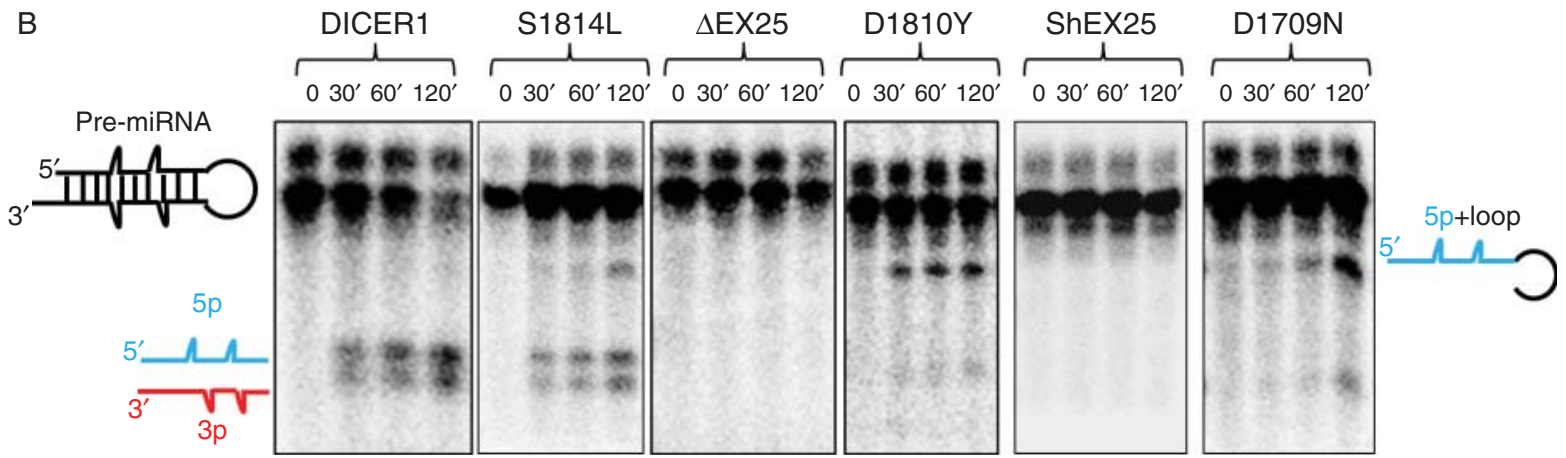

Figure 2

Analysis of the effect of DICER 1 mutations c.5441C $>$ T and c.5428G $>$ T on exon skipping and pre-miR122 cleavage. (A) Exon cassette experiments demonstrate that c.5441C $>$ T can cause exon skipping and c.5428G $>$ T and can create a cryptic splice site. ** represents skipped exon $25, * * *$ represents

elsewhere have also been evaluated using our in vitro cleavage assay. The variant p.S1814L has reduced 5p and $3 p$ miRNA generation and produces some $5 p$ miRNA + loop (5p+loop), as compared to a normal DICER1 protein (Fig. 2B). This decrease in both $5 \mathrm{p}$ and $3 \mathrm{p}$ miRNAs is somewhat unexpected given that the mutation is situated in the RNase IIIb domain. This particular substitution could compromise access to the RNase IIIa/b domain by altering protein folding (Supplementary Figure 4). We postulate that the effect of incomplete miRNA generation on transcript silencing is dependent on what species of miRNA is loaded into the RNA induced silencing complex (RISC). For example, off target silencing could occur if either $5 p+$ loop or $3 p$ is loaded instead of $5 p$. Alternatively, no silencing might result if neither $5 p+$ loop nor $3 p$ is loaded. In contrast, the predicted protein p. $\Delta \mathrm{EX} 25$ does not produce either $5 \mathrm{p}$ or $3 \mathrm{p}$ miRNAs (Fig. 2B).

Sanger sequencing of the region encoding the DICER1 RNase III domains in gDNA extracted from the formalin exon 25 bearing c.5441C $>$ T, $€$ represents exon 25 with $5428 \mathrm{G}>\mathrm{T}$, and $¥$ represents cryptic splice site. (B) In vitro cleavage time course demonstrate the effect of missense mutations on pre-miR122 processing. A full colour version of this figure is available at http://dx.doi.org/10.1530/ERC-15-0460.

fixed paraffin embedded (FFPE) ovarian tumor revealed the somatic mutation, c. $5125 \mathrm{G}>\mathrm{A}$, which is predicted to encode p.D1709N (Fig. 1B and C). We were unable to determine phase of germ-line and somatic mutation in the ovarian tumor. We repeated the in vitro cleavage assay using this mutation. As previously reported (Heravi-Moussavi et al. 2012), this mutation renders the resulting DICER1 protein incapable of producing $5 \mathrm{p}$ miRNAs and causes the accumulation of a $5 \mathrm{p}+$ loop species (Fig. 2B). Given the finding of a somatic DICER1 mutation in the ovarian tumor, the initial diagnosis of adult GCT was questioned, since these neoplasms do not appear to be part of the DICER1 syndrome (Foulkes et al. 2014). Following pathological review, the histopathological diagnosis was revised to an SLCT of intermediate grade with a minor heterologous component of hepatoid cells (Supplementary Figure 2A, B, C, D, E and F). The hepatoid areas showed focal positivity for AFP by immunohistochemistry (not shown) which may explain the proband's elevated

Published by Bioscientifica Ltd 
AFP levels. The tumor tested negative for the presence of the c.402C $>$ G FOXL2 mutation known to be present in almost all adult GCTs (Shah et al. 2009), further supporting the diagnosis of SLCT over adult GCT.

Sanger sequencing of the RNase III-encoding exons in gDNA extracted from a FFPE MNG sample revealed a somatic DICER1 mutation, c.5428G $>\mathrm{T}$, which is predicted to encode p.D1810Y (Fig. 1B-C). To our knowledge, this is the first report of a somatic DICER1 mutation in an MNG. Moreover, we were able to show that the germ-line (c.5441C $>\mathrm{T}$ ) and somatic (c.5428G $>\mathrm{T}$ ) mutations were in trans (Supplementary Figure 7, see section on supplementary data given at the end of this article) and thus the 'two-hit' paradigm is fulfilled for MNG pathogenesis. In silico analysis of the c. $5428 \mathrm{G}>\mathrm{T}$ mutation predicted an increased chance of exon 25 skipping (EX-SKIP (Raponi et al. 2011) denoted by $€$ in Fig. 2A, right panel) and the creation of a cryptic splice site (BDGP (Reese et al. 1997) $¥$ in Fig. 2A, right panel, and Supplementary Figure 8). In vitro exon cassette assay suggests that c. $5428 \mathrm{G}>\mathrm{T}$ does create a cryptic splice site (Fig. 2A, $¥$ ), which is predicted to be translated to a shorter protein, p.K1844fsX17, here abbreviated to ShEX25. The ShEX25 DICER1 protein cannot produce $5 \mathrm{p}$ or $3 \mathrm{p}$ miRNAs (Fig. $2 \mathrm{~B}$ ). However, c.5428G $>\mathrm{T}$ may also behave as a missense mutation (p.1810Y). In this case, it has a defect in 5p miRNA generation (Fig. 2B).

The most common mechanism by which tumorigenesis occurs in the DICER1 syndrome (OMIM 601200) is germ-line inactivation of one DICER 1 allele followed by one somatically acquired mutation, occurring on the remaining allele, which affects exons encoding the RNase IIIb domain. This combination results in a decrease in 5p miRNA generation in susceptible initiating cells at a critical time during tissue development, which promotes lesion formation (Foulkes et al. 2014). The two hits we observed in the SLCT are consistent with these findings (Fig. 2B). It is notable that we observed the same two-hit phenomenon in the MNG (Supplementary Figure 7), as goiter can be either monoclonal or polyclonal in nature (Kopp et al. 1994). Moreover, MNG is not classified by the World Health Organization as a neoplasm, but as a benign hyperplasia (Horowitz et al. 2004). Further studies of DICER1 related goiters are warranted.

This report documents the occurrence of a novel germline DICER1 single base pair substitution (c.5441C $>$ T) localized to within the RNase IIIb domain in a patient with two conditions clearly associated with the DICER1 syndrome. We consider this mutation deleterious because i) it has not been reported before (ExAC, EVS, ClinVar), ii) it lies one residue away from the metal ion binding residue 1813 , and iii) has a defect in miRNA processing. The clinical phenotype, however, associated with this mutation appears to be somewhat milder than the very severe phenotypes that are associated with either germline or mosaic RNase IIIb mutations that directly impinge on metal ion binding residues (de Kock et al. 2014, 2015, Klein et al. 2014), emphasizing the need for full characterization of previously unreported DICER 1 variants. This requires a combined approach involving pathology, genetics, biochemistry, and molecular modeling. After the accepted preprint of this paper was published on-line, Rutter et al. (2015) reported a family with 6 members with differentiated thyroid cancer, MNG, and SLCT and complete segregation of the same c.5441C > T; p.S1814L germline mutation we report here. This finding strongly supports our conclusion that this mutation is pathogenic.

Using diverse methods, we have demonstrated that the three DICER1 missense mutations studied here can have distinct effects on miRNA processing, which may be mediated by differing effects of the single base pair changes on the DICER1 mRNA and protein. Thus, c.5125G >A functions as a missense mutation, leading to loss of $5 p$ miRNA, whereas c.5441C $>\mathrm{T}$, functioning as a missense mutation, affects both $5 \mathrm{p}$ and $3 \mathrm{p}$ miRNA generation in vitro and c.5428G $>\mathrm{T}$ can generate a cryptic splice site. We also report the first instance of biallelic DICER1 mutations in a MNG. Further, the identification of a somatic DICER1 mutation in the ovarian tumor prompted review of the original pathology, further illustrating the use of adjunct molecular analysis in the diagnosis of uncommon and histologically problematic ovarian neoplasms. Future studies to characterize individual novel missense mutations will need to include cell and/or animal models.

$$
\begin{array}{r}
\text { M K Wu }{ }^{1} \\
\text { L de Kock }^{1} \\
\text { L S Conwell } \\
\text { C J R Rtewart } \\
\text { B R King } \\
\text { 6,7 } \\
\text { C S Choong } \\
\text { K Hussain } \\
\text { 10 } \\
\text { N Sabbaghian } \\
\text { I J MacRae }^{11} \\
\text { M R Fabian } \\
\text { W D Foulkes } \\
\text { W D Foul13 }
\end{array}
$$

${ }^{1}$ Department of Medical Genetics, Lady Davis Institute Jewish General Hospital, McGill University, Montréal, Quebec, Canada

Published by Bioscientifica Ltd. 
${ }^{2}$ Department Endocrinology and Diabetes, Lady Cilento Children's Hospital, Brisbane, Queensland, Australia

${ }^{3}$ School of Medicine, University of Queensland, Brisbane,

Queensland, Australia

${ }^{4}$ Queensland Children's Medical Research Institute, Brisbane,

Queensland, Australia

${ }^{5}$ Department of Histopathology, King Edward Memorial Hospital, Perth, Australia

${ }^{6}$ John Hunter Children's Hospital, Lookout Road, Newcastle, New South Wales, Australia

${ }^{7}$ School of Medicine and Public Health, Hunter Medical Research Institute, University of Newcastle, Rankin Park,

New South Wales, Australia

${ }^{8}$ School of Paediatrics and Child Health, University of Western Australia, Crawley, Western Australia, Australia

${ }^{9}$ Department of Paediatric Endocrinology and Diabetes, Princess Margaret Hospital for Children, Child and Adolescent Health Service, Subiaco, Western Australia, Australia

${ }^{10}$ Genetics and Epigenetics in Health and Disease Genetics and Genomic Medicine Programme UCL Institute of Child Health Great Ormond Street Hospital for Children, London, UK

${ }^{11}$ The Scripps Research Institute, 3215 Merryfield Row, San Diego, California, USA

${ }^{12}$ Departments of Oncology and Experimental Medicine, McGill University, Montréal, Quebec, Canada

${ }^{13}$ Program in Cancer Genetics, Departments of Oncology and Human Genetics, McGill University, Montréal, Quebec, Canada, H3T 1E2

(Correspondence should be addressed to W D Foulkes; email: william.foulkes@mcgill.ca)

\section{Supplementary data}

This is linked to the online version of the paper at http://dx.doi.org/10.1530/ ERC-15-0460.

\section{Declaration of interest}

The authors declare that there is no conflict of interest that could be perceived as prejudicing the impartiality of the research reported.

\section{Funding}

M K Wu is a recipient of an Fonds de Recherche du Québec-Santé (FRQS) scholarship. L de Kock is a recipient of the Vanier Canada Graduate Scholarship. This work was supported by Alex's Lemonade Stand Fund (awarded to W D Foulkes), and by the Canadian Institutes of Health Research (MOP-130425) (to M R Fabian).

\section{Acknowledgements}

We thank the University of Exeter Molecular Genetics Laboratory for $A B C C 8$ genetic testing and Dr I Bah for images of the MNG.

\section{References}

Foulkes WD, Priest JR \& Duchaine TF 2014 DICER1: mutations, microRNAs and mechanisms. Nature Reviews. Cancer 14 662-672. (doi:10.1038/nrc3802)

Heravi-Moussavi A, Anglesio MS, Cheng SW, Senz J, Yang W, Prentice L, Fejes AP, Chow C, Tone A, Kalloger SE et al. 2012 Recurrent somatic DICER1 mutations in nonepithelial ovarian cancers. New England Journal of Medicine 366 234-242. (doi:10.1056/NEJMoa1102903)

Horowitz B, Minor P, Morgenthaler JJ, Burnouf T, McIntosh R, Padilla A, Thorpe R \& van Aken WG 2004 WHO expert committee on biological standardization. World Health Organization Technical Report Series 924 $1-232$.

Klein S, Lee H, Ghahremani S, Kempert P, Ischander M, Teitell MA, Nelson SF \& Martinez-Agosto JA 2014 Expanding the phenotype of mutations in DICER1: mosaic missense mutations in the RNase IIIb domain of DICER1 cause GLOW syndrome. Journal of Medical Genetics 51 294-302. (doi:10.1136/jmedgenet-2013-101943)

de Kock L, Sabbaghian N, Plourde F, Srivastava A, Weber E, Bouron-Dal Soglio D, Hamel N, Choi JH, Park SH, Deal CL et al. 2014 Pituitary blastoma: a pathognomonic feature of germ-line DICER1 mutations. Acta Neuropathologica 128 111-122. (doi:10.1007/s00401-014-1285-z)

de Kock L, Wang YC, Revil T, Badescu D, Rivera B, Sabbaghian N, Wu M, Weber E, Sandoval C, Hopman SM et al. 2015 High-sensitivity sequencing reveals multi-organ somatic mosaicism causing DICER1 syndrome. Journal of Medical Genetics [in press]. (doi:10.1136/ jmedgenet-2015-103428)

Kopp P, Kimura ET, Aeschimann S, Oestreicher M, Tobler A, Fey MF \& Studer H 1994 Polyclonal and monoclonal thyroid nodules coexist within human multinodular goiters. Journal of Clinical Endocrinology and Metabolism 79 134-139. (doi:10.1210/jcem.79.1.7517946)

Nessa A, Aziz QH, Thomas AM, Harmer SC, Tinker A \& Hussain K 2015 Molecular mechanisms of congenital hyperinsulinism due to autosomal dominant mutations in ABCC8. Human Molecular Genetics 24 5142-5153. (doi:10.1093/hmg/ddv233)

Raponi M, Kralovicova J, Copson E, Divina P, Eccles D, Johnson P, Baralle D \& Vorechovsky I 2011 Prediction of single-nucleotide substitutions that result in exon skipping: identification of a splicing silencer in BRCA1 exon 6. Human Mutation 32 436-444. (doi:10.1002/humu.21458)

Reese MG, Eeckman FH, Kulp D \& Haussler D 1997 Improved splice site detection in Genie. Journal of Computational Biology 4 311-323. (doi:10.1089/cmb.1997.4.311)

Rutter MM, Jha P, Schultz KA, Sheil A, Harris AK, Bauer AJ, Field AL, Geller J $\&$ Hill DA 2015 DICER1 mutations and differentiated thyroid carcinoma: evidence of a direct association. Journal of Clinical Endocrinology and Metabolism [in press]. (doi:10.1210/jc.2015-2169)

Shah SP, Kobel M, Senz J, Morin RD, Clarke BA, Wiegand KC, Leung G, Zayed A, Mehl E, Kalloger SE et al. 2009 Mutation of FOXL2 in granulosa-cell tumors of the ovary. New England Journal of Medicine $\mathbf{3 6 0}$ 2719-2729. (doi:10.1056/NEJMoa0902542)

Takeshita D, Zenno S, Lee WC, Nagata K, Saigo K \& Tanokura M 2007 Homodimeric structure and double-stranded RNA cleavage activity of the C-terminal RNase III domain of human dicer. Journal of Molecular Biology 374 106-120. (doi:10.1016/j.jmb.2007.08.069)

Received 30 October 2015

Accepted 6 November 2015

Made available online as an Accepted Preprint

6 November 2015

Published by Bioscientifica Ltd. http://erc.endocrinology-journals.org DOI: $10.1530 / E R C-15-0460$
(C) 2016 Society for Endocrinology Printed in Great Britain 